\title{
Invasive Lobular Carcinoma within a Fibroadenoma: A Case Report and Review of the Literature
}

\author{
Dimitrios Kokkonis, Mohamed Razick Sait, Anne Thorpe, Ralia Bunza, Rajiv Vashisht, \\ Musa Barkeji
}

Department of Breast Surgery, West Middlesex University Hospital, London, United Kingdom

Email address:

dimitrioskokkonis77@gmail.com (D. Kokkonis)

\section{To cite this article:}

Dimitrios Kokkonis, Mohamed Razick Sait, Anne Thorpe, Ralia Bunza, Rajiv Vashisht, Musa Barkeji. Invasive Lobular Carcinoma within a Fibroadenoma: A Case Report and Review of the Literature. Journal of Surgery. Vol. 7, No. 2, 2019, pp. 35-37. doi: 10.11648/j.js.20190702.12

Received: February 18, 2019; Accepted: April 9, 2019; Published: May 6, 2019

\begin{abstract}
Fibroadenoma (FAD) is the most common type of breast lesion especially in young female individuals. Although malignant transformation in FAD is rare, several reports have described a higher risk of subsequent breast carcinoma amongst patients who have FAD with specific histologic features. The case of a 30-year-old female exhibiting invasive lobular carcinoma arising within an excised fibroadenoma is being presented. The literature was reviewed with regards to the increased likelihood of developing breast cancer in patients with FADs. The incidence of cancer found in an excised FAD is extremely low, but there are specific proliferative changes on the histology after a core needle biopsy, that they should warrant excision biopsy.
\end{abstract}

Keywords: Fibroadenoma, Invasive Lobular Cancer, Core Biopsy, Excision Biopsy

\section{Introduction}

Fibroadenoma (FAD) is a common benign disease of the breast in females. Malignant transformation in FAD is rare. However, there are reports describing a higher risk of subsequent breast cancer amongst patients who have FAD with relative risks (RRs) ranging from 1.48 to 1.7 [1-5].

FAD may occur at any age, however is mostly encountered in premenopausal women and the peak age of incidence is the second and third decade of life [6]. On histology FAD is a benign tumour composed of both epithelial and stromal components. They can be classified as simple or complex according to specific histological features [7]. Complex FADs are associated with histologic characteristics, including cysts ( $>3 \mathrm{~mm})$, sclerosing adenosis, epithelial calcifications, or papillary apocrine metaplasia [1]. FADs resemble a hyperplastic breast lobule and they respond to same hormonal stimuli as the normal breast tissue [8]. In the past, FADs were acknowledged as benign tumours of the breast [9] and traditionally were treated in their entity with surgical excision to exclude malignancy. That management has been challenged, as in most of the cases a confident diagnosis and exclusion of malignancy is possible with the combination of imaging techniques and needle core biopsy. Also, the current view that FADs are hyperplastic rather than neoplastic and that they represent an aberration of normal lobular hypertrophy during the period of maximal estrogen exposure supports a policy of non-surgical management [10-11]. However, symptomatic, progressively enlarging masses or atypical presentations may warrant surgical excision. Although very rare, breast cancer arising within fibroadenoma can still be found. The incidence of the above ranges from $0.02 \%$ to $0.125 \%$, according to different reports $[1,7,12]$. So far, just over 100 cases have been reported in the literature $[6,13,14]$.

\section{Case Report}

A 30-year-old lady represented in the breast clinic with a 6 years history of left breast FAD, which had increased in size with no pain, skin changes or nipple discharge. She was seen initially 6 years ago, when she had a core needle biopsy suggestive of a left breast FAD.

On examination, there was a $5 \times 6 \mathrm{~cm}$ solid, mobile lump in the upper outer quadrant of the left breast. Examination of the contralateral breast was normal.

Ultrasound scan revealed a $4.1 \mathrm{~cm}$ - previously $1.7 \mathrm{~cm}-$ hypoechoic mass at 2 o'clock position of the left breast, with some internal vascularity, likely to represent a FAD.

$4 \times 14$ gauge core biopsies were obtained, as it had increased significantly in size since the last time. The histology again 
confirmed a FAD (B2).

MDT (Multi Disciplinary Team) recommendation was for excision considering the significant increase in size. Excision biopsy revealed a $19 \mathrm{gr}$ lobulated mass, $3.5 \mathrm{~cm}$ in maximum diameter, compatible with FAD. Unexpectandly, within the $\mathrm{FAD}$, there was a component of invasive lobular carcinoma measuring $15 \mathrm{~mm}$ in maximum diameter and associated lobular carcinoma in situ (LCIS). As the tumour was confined within the FAD, resection margins were all clear. No lymphovascular permeation. The receptor status on immunohistochemistry revealed Oestrogen receptors (ER) positive and HER 2 negative. The specimen was reviewed by 2 different consultant histolpathologists who concurred with the findings.

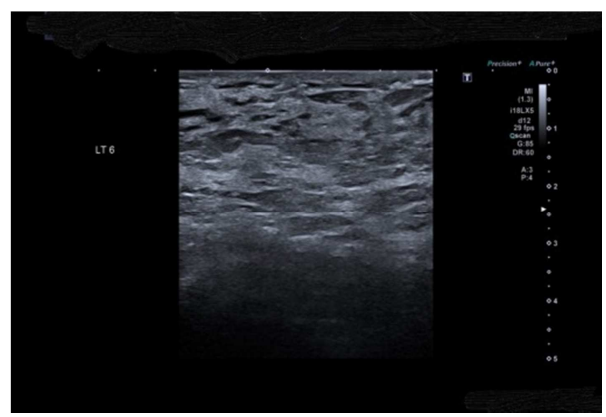

Figure 1. Most recent USS showing FAD of left breast.

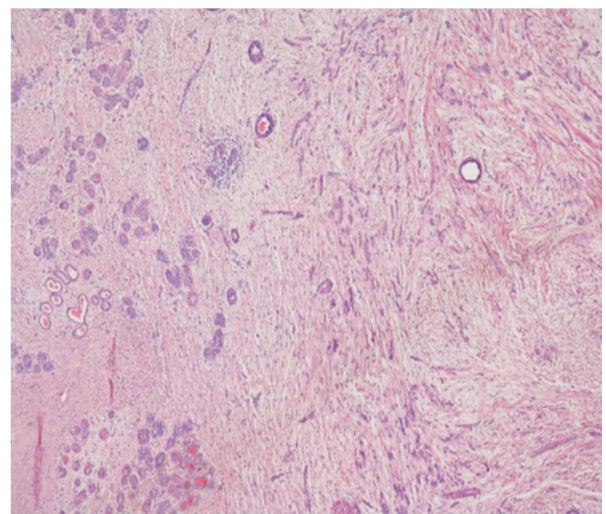

Figure 2. Histopathology image showing both FAD and invasive cancer.

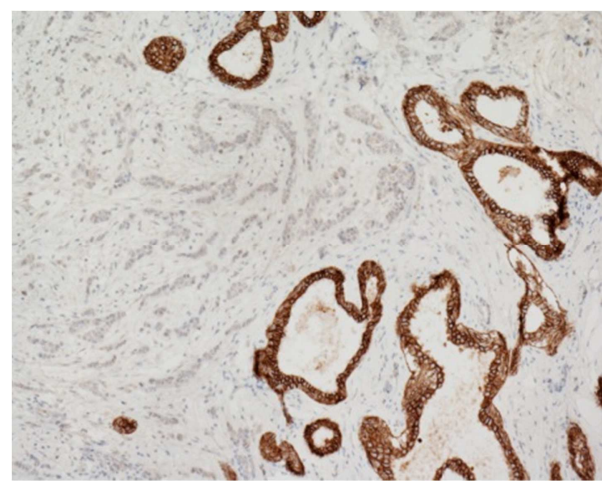

Figure 3. Different stain of histopathology showing FAD and the cancer.

A contrast enhanced MRI scan revealed no concurrent lesions on either breasts.

She subsequently underwent excision of cavity walls and sentinel lymph node biopsy (SLNB), to rule out metastasis in regional lymph nodes. Cavity wall biopsies and SLNB confirmed no malignancy.

The patient received adjuvant radiotherapy to the breast and was commenced on tamoxifen $20 \mathrm{mg}$.

She is being followed up with regular annual surveillance mammograms and scans.

\section{Discussion}

FAD is a common type of benign breast lesion with a reported incidence rate of $7-13 \%$ in female individuals and commonly in the second and third decades of life [6]. A carcinoma arising in a FAD is extremely rare, with an incidence of $0.02 \%$ to $0.125 \%[1,7,12]$. The mean age of patients found to have cancer (in situ or invasive) within a FAD - is 42-44 years, approximately 20 years subsequent to the peak age of occurrence of FAD [15]. Most breast cancers found in FADs are reported to be in situ $(66.9 \%$ LCIS and $12.4 \%$ DCIS), followed by invasive carcinoma ( $11 \%$ IDC and $3.4 \%$ ILC) [16]. Given the extremely low incidence of ILC found in FAD, the current case report is one of the rarest.

Dupont et al [1] described breast cancer in women with FAD more than two decades ago. He concluded that FAD confers an overall relative risk (RR) of $2.17 \%$. However the risk increased to $3.1 \%$ for women with complex FAD. He further showed that the increased risk persisted for decades after the initial diagnosis. In a recent Cohort Study conducted in Mayo Clinic [17], the risk was not significantly different between women with simple FAD and women with no FAD. It is notable that these 2 cohorts are amongst the largest cohorts of women with FAD studied to date. The data from the above studies suggest that the risk of breast cancer in women with FAD is driven by the histologic features present in association with the FAD, such as proliferative disease without atypia (PDWA) or atypical hyperplasia (AH). PDWA criteria were ductal hyperplasia (more than mild), papillomas, radial scar or sclerosing adenosis. AH included atypical ductal or lobular hyperplasia [17]. In the institution, where the current patient was treated, the above features, if found in a lesion after a needle core biopsy, are characterized as B3 lesions (Breast Pathology Coding system in UK) and patients are offered an excision biopsy.

Neville G et al [18] suggested that excision based solely on size is not warranted in clinically and radiologically concordant cases with a diagnosis of FAD on CNB. Their study included a total of 12,109 consecutive radiologically guided CNB that were performed in a period of time of 5 years; 3438 with a diagnosis of FAD were identified of which 290 cases went on to have surgical excision. Of those 290 cases; $98.28 \%(n=285)$ were confirmed as FAD on excision. The remaining $1.72 \%(\mathrm{n}=$ 5) had atypical features-FAD with LCIS $(n=1)$, benign PT $(n=$ $3)$, and invasive ductal carcinoma $(\mathrm{n}=1)$.

In this case, the patient received Breast Conserving Surgery (BCS), as the final pathology report confirmed clear margins $(>2 \mathrm{~mm})$. In a recent collective analysis of case reports in the literature regarding breast carcinomas arising within FADs 
[19], most of the patients received BCS. Breast carcinomas are usually found incidentally during histology, when the tumour is already excised. This could explain the high rate $(66.7 \%)$ of BCS in these patients. The BCS rate is compatible with the reported data in the management of breast cancer with the same tumour size in the NASBP B-06 trial (National Surgical Adjuvant Breast and Bowel Project) [19].

Our patient received adjuvant radiotherapy and endocrine blockade, as it would be the standard management in any patient diagnosed with invasive cancer which was ER receptor positive. Tiu et al. stated that breast cancer found within a FAD behaves like breast cancer arising de novo and hence the treatment would remain the same [20].

In author's institution, simple fibroadenomas (B2) $<3.5 \mathrm{~cm}$ are being managed conservatively. Criteria to proceed with excision biopsy are FADs bigger than $3.5 \mathrm{~cm}$ or biopsy containing atypia or proliferative features that characterize it as B3 lesion, or the patient is symptomatic or wishes to have it excised.

\section{Conclusion}

Malignant transformation in FAD is rare. There is general consensus that the management of FAD depends on the presence of proliferative changes. There should be an open discussion with patients with certain features on biopsy of FAD and they should be offered excision and if incidentally noted to have cancer should be managed as per standard protocol.

\section{References}

[1] Dupont W, Page D, Pari F, Vnencak-Jones C, Plummer W, Rados M Schyler. Long-Term Risk of Breast Cancer in Women with Fibroadenoma. N Engl J Med 1994; 331(1):10-15.

[2] Kuijper A, Mommers EC, van der Wall E, van Diest PJ. Histopathology of fibroadenoma of the breast. Am J Clin Pathol 2001; 115(5):736-742.

[3] McDivitt RW, Stevens JA, Lee NC, Wingo PA, Rubin GL, Gersell D. The Cancer and Steroid Hormone Study Group. Histologic types of benign breast disease and the risk for breast cancer. Cancer 1992; 69(6):1408-1414.

[4] Kabat GC, Jones JG, Olson N, et al. A multi-center prospective cohort study of benign breast disease and risk of subsequent breast cancer. Cancer Causes Control 2010; 21(6):821-828.

[5] El-Wakeel H, Umpleby HC. Systematic review of fibroadenoma as a risk factor for breast cancer. Breast 2003; 12(5):302-307.

[6] Bin Hua, Jing-Yong Xu, Lei Jiang, Zheng Wang. Fibroadenoma with an unexpected lobular. carcinoma in situ: a case report and review of the literature. Oncology Letters 1 2015; 1397-1401.
[7] Buzanowski-Konakry K, Harrison EG Jr and Payne WS: Lobular carcinoma arising in fibroadenomas of the breast. Cancer 1975; 35:450-456.

[8] Martin PM, Kutten F, Serment H, Jarvis MP. Studies on clinical, hormonal and pathological correlations in breast fibroadenomas. J Steroid Biochem 1978; 9:1251-2.

[9] World Health organization Histological typing of breast tumours. $2^{\text {nd }}$ ed. Geneva: WHO 1981.

[10] Hughes LE, Mansel RE, Webster DJT, Aberration of normal development and involution: a new perspective on pathogenesis and nomenclature of benign breast disorders. Lancet 1987; 11:1316-9.

[11] Carty N J, Carter C, Rubin C, Ravichandran, Royal GT. Taylor I. Management of fibroadenoma of the breast. Ann R Coll Surg Engl 1995; 77:127-30.

[12] Deschenes L, Jacob, Fabia J, Christen A: Beware of breast fibroadenomas in middle-aged wome. Can j Surg 1985; 28:372-374.

[13] Iyengar KR, Peh SC, Yip CH and Vijayananthan A: Infiltrating duct carcinoma within a fibroadenoma. Indian J Cancer 2009; 46:244-246.

[14] Abe H, Hanasawa K, Naitoh H, et al: Invasive ductal carcinoma within a fibroadenoma of the breast. Int J Clin Oncol 2004; 9: 334-338.

[15] Pick PW and Iossifides IA: Occurrence of breast carcinoma within a fibroadenoma. A review. Arch Pathol Lab Med 1984; 108:590-594.

[16] Fukuda M, Nagao K, Nishimuta R, Matsuda M, Baba K, Ueno Y, Morinaga $\mathrm{H}$, Omachi $\mathrm{H}$, Hamada T: Carcinoma arising in fibroadenoma of the breast- a case report and review of the literature. Jpn J Surg 1989; 19:593-596.

[17] Aziza Nassar, Daniel W., Visscher, Amy C. Degnim, Messr. Ryan D. Frank, Messr. Robert A. Vierkant, Marlene Frost, Derek C. Radisky, Celine Vachon, Ruth A. Kraft, Lynn C. Hartmann, Karthik Ghosh. Complex Fibroadenoma and Breast Cancer Risk: Mayo Clinic Benign Breast Disease Cohort Study. Breast Cancer Res Treat 2015; 153(2): 397-405.

[18] Neville G, Neill CO, Murphy R, Corrigan M, Redmond PH, Feeley L, Bennett MW, O'Connell F, Browne TJ. Is excision biopsy of fibroadenomas based solely on size criteria warranted? Breast J 2018 May; 25. doi: 10.1111/tbj.13069.

[19] Yu Ting Wu, Shou-Tung Chen, Chi-Jung Chen, Yao-Lung Kuo, Ling-Ming Tseng, Dar-Ren Chen, Shou-Jen Kuo, Hung-Wen Lai. Breast cancer arising within fibroadenoma: collective analysis of case reports in the literature and hints on treatment policy. World Journal of Surgical Oncology 2014; 12:335-38.

[20] Tiu CM, Chou YH, Chiou SY, Hsu CY, Chen SP, Chiang HR, Lai CR, Tseng LM, Wang HK, Chiou HJ: Development of a carcinoma in situ in a fibroadenoma: color Doppler sonographic demonstration. J Ultrasound Med 2006; 25:1335-1338. 